\title{
A COVID-19 infection risk model for frontline health care workers
}

\author{
Louie Florendo Dy ${ }^{1,2} \cdot$ Jomar Fajardo Rabajante ${ }^{3,4}$ (C)
}

Received: 15 April 2020 / Revised: 5 July 2020 / Accepted: 9 July 2020 / Published online: 8 August 2020

(c) Springer-Verlag GmbH Austria, part of Springer Nature 2020

\begin{abstract}
The number of confirmed COVID-19 cases admitted in hospitals is continuously increasing in the Philippines. Frontline health care workers are faced with imminent risks of getting infected. In this study, we formulate a theoretical model to calculate the risk of being infected in health care facilities considering the following factors: the average number of encounters with a suspected COVID-19 patient per hour; interaction time for each encounter; work shift duration or exposure time; crowd density, which may depend on the amount of space available in a given location; and availability and effectiveness of protective gears and facilities provided for the frontline health care workers. Based on the simulation results, a set of risk assessment criteria is proposed to classify risks as 'low', 'moderate', or 'high'. We recommend the following: (1) decrease the rate of patient encounter per frontline health care worker, e.g., maximum of three encounters per hour in a 12-h work shift duration; (2) decrease the interaction time between the frontline health care worker and the patients, e.g., less than 40 min for the whole day; (3) increase the clean and safe space for social distancing, e.g., maximum of $10 \%$ crowd density, and if possible, implement compartmentalization of patients; and/or (4) provide effective protective gears and facilities, e.g., 95\% effective, that the frontline health care workers can use during their shift. Moreover, the formulated model can be used for other similar scenarios, such as identifying infection risk in public transportation, school classroom settings, offices, and mass gatherings.
\end{abstract}

Keywords COVID-19 $\cdot$ SARS-CoV-2 $\cdot$ Philippines $\cdot$ Healthcare $\cdot$ Crowd dynamics $\cdot$ Frontliner

\section{Introduction}

As of March 20, 2020, Coronavirus Disease (COVID-19) has infected 250,704 worldwide, resulting in 10,256 deaths, with Italy surpassing China in the reported number of deaths on the same day (Novel Coronavirus 2020; Italy Coronavirus Death Toll Overtakes China 2020). Aggressive suppression strategies have been recommended (Ferguson et al. 2020),

Jomar Fajardo Rabajante

jfrabajante@up.edu.ph

Louie Florendo Dy

1fdy@up.edu.ph

1 College of Medicine, University of the Philippines Manila, Manila, Philippines

2 Philippine General Hospital, University of the Philippines, Manila, Philippines

3 Institute of Mathematical Sciences and Physics, University of the Philippines Los Baños, Los Baños, Philippines

4 Faculty of Education, University of the Philippines Open University, Los Baños, Philippines and countries across the world have implemented strategies to mitigate the damage caused by this pandemic (Anderson et al. 2020).

Health care workers work in the frontlines across the world unceasingly, running the greatest risk of getting infected and infecting others in their immediate environment—in the hospital, at home-or wherever they go. In the Philippine context, health care system can be described as two tiered (Dayrit et al. 2018). There is a huge disparity in the capacity between the public and private health sectors (Dayrit et al. 2018). As of 2016, there are a total of 101,688 hospital beds, with a ratio of 23 beds for 10,000 people in the National Capital Region, and more than half (53.4\%) of these are in private hospitals (Dayrit et al. 2018). The number of confirmed COVID-19 cases admitted in hospitals is continuously increasing exponentially (Panela 2020). Therefore, given that the health system is likely to be overwhelmed (Dayrit et al. 2018; Baticulon 2020), these frontline health care workers (frontliners) are faced with unimaginable risks of getting infected. 
Every doctor, nurse, medical technologist, radiation technologist, nursing assistant, hospital janitor and security guard will inevitably face the risk of COVID-19 infection. Here, we formulate a mathematical model to investigate how many frontliners are expected to be infected under certain scenarios (Rabajante 2020). We use this expected number of possible new infections as a measure of the risk. A set of risk assessment criteria has been formulated based on the theoretical results to determine if a frontliner has low, moderate or high risk of COVID-19 infection.

Mathematical models can be used for predicting scenarios and in prescribing solutions to problems (Rabajante 2020; Choudhury et al. 2018; Ferrett et al. 2020; Cortez 2017), such as addressing the COVID-19 pandemic. Based on the simulations and risk assessment, several recommendations are suggested to inhibit the spread of SARS-CoV-2, especially in health care facilities.

Average number of encounters per hour $\approx$

Average number of patients encountered per hour $\times \frac{\text { Average duration of interaction in minutes }}{0.5 \text { minutes }}$.

\section{Results and discussion}

The formulated risk model (see "Appendix: Methods") aims to examine the risk factors of virus transmission per day, quantify these risks, estimate the number of new infections, and suggest ways to minimize these risks. There are several factors that determine the risk of infection:

- Average number of COVID-19 patients (or, in other settings, number of susceptible persons) entering a given location at a given time, whether they are confirmed to be positive or not;

- Average number of encounters with a patient (or, in other settings, any susceptible person) at a given time, whether COVID-19 infected or not, where an encounter is defined to be less than or equal to $30 \mathrm{~s}$;

- Duration of interaction of each of these encounters;

- Work shift duration of each frontliner (or, in other settings, can be equivalent to the exposure time for any person in public transportation, offices, classrooms, and mass gatherings);

- Crowd density, which may depend on the amount of space available in a given location, the presence of compartments or dividers in a room, and how frequent cleaning is done in the environment as the density of SARSCoV-2 virus particles present on surfaces limits the safe space available; and

- Level of protection present (e.g., isolation booths, N95 masks, face and eye shields) including level of protec- tion to reduce exposure to aerosolized particles (e.g., for those tasked to do intubation either via direct or via video laryngoscopy, to do nasopharyngeal or oropharyngeal swabs).

In the following discussion, the parameters will be discussed in terms of the frontliner setting, but the parameters can likewise be applied in other settings, such as in crowded places, classrooms, offices, public vehicles, and markets.

\subsection{Average number of encounters and work shift duration (exposure time)}

The average number of encounters per hour can be defined as the average number of patients a frontliner has interacted in an hour given that an interaction is less than or equal to $30 \mathrm{~s}$. We can convert number of patients per minute to number of encounters using the following formula:

For example, a $10-15$-min interview with a patient is equivalent to 20-30 encounters. A doctor doing brief rounds on 10 patients (where each patient is looked upon in at most $30 \mathrm{~s}$ ) or the situation of having 5 patients per minute are equivalent to 10 encounters.

According to our simulation results, the expected number of infected frontliners increases as the average number of encounters between the frontliner and COVID-19 patients increases as well as when work shift duration or exposure time increases (Fig. 1a, b). If there is highly interacting population (e.g., the average number of encounters per hour is 120 , which means that the frontliners and the patients are interacting every $30 \mathrm{~s}$ ) or a series of long interactions (e.g., 4 patient interviews per hour where each interview takes $15 \mathrm{~min}$ ), then there is a high chance that one person will be infected. If there is low interaction rate (e.g., seeing one patient only for less than or equal to $30 \mathrm{~s}$ once per hour), then the chance of getting infected is low but the risk is not zero.

It should be noted that if the number of possible infected frontliners is greater than or equal to one, then there is high risk of infection; if the number of possible infected frontliners is less than one but not equal to zero, then there is still some level of risk (low or moderate risk of infection). In a 7-h work shift duration, there is high chance a frontliner will be infected if the interaction rate is around 12 encounters per hour (Fig. 1b). This can be imagined as a triage nurse seeing 1 patient for at most $30 \mathrm{~s}$ every $5 \mathrm{~min}$ during the duration of his or her 7-h work shift. 

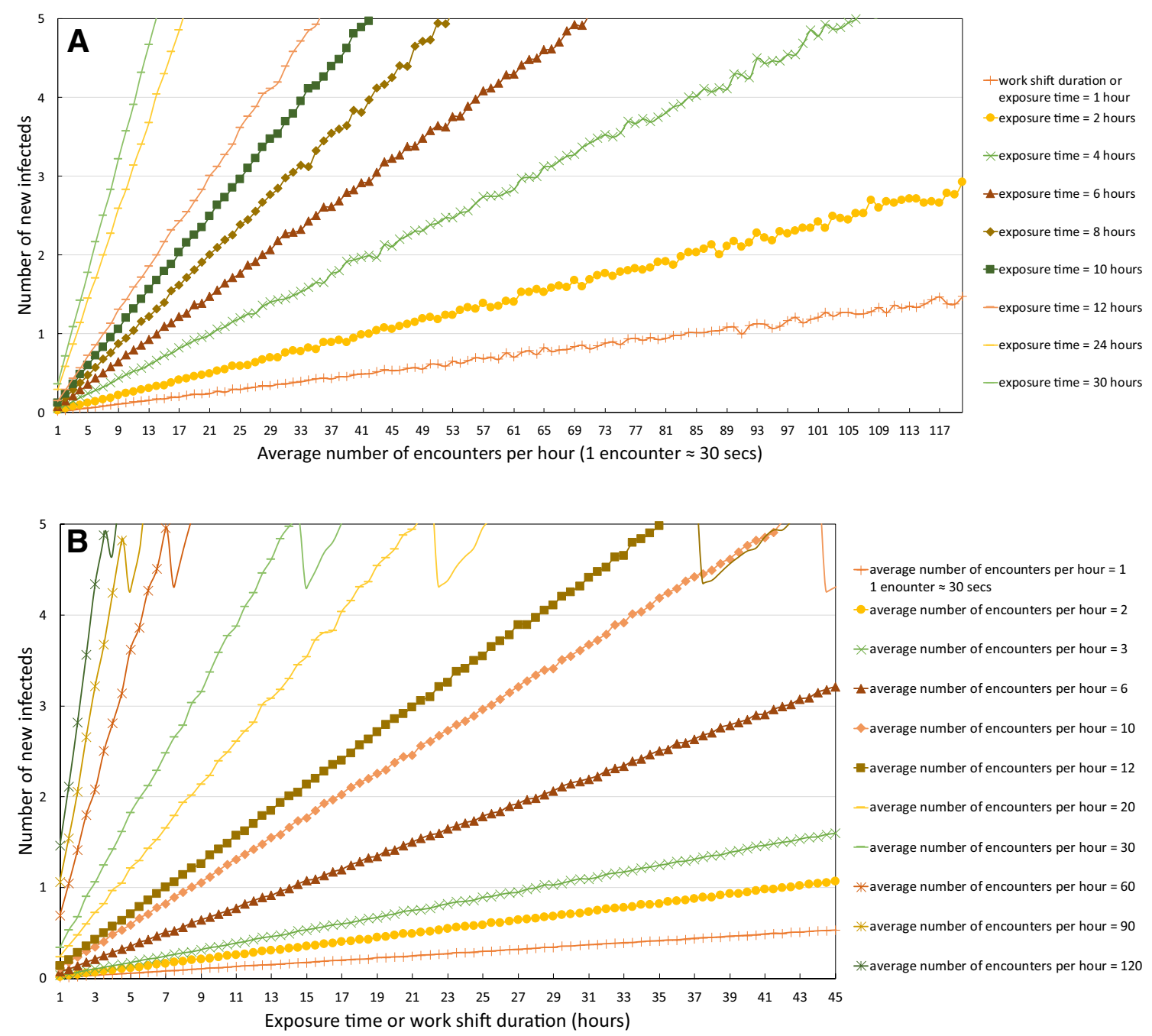

Fig. 1 Relationship of work shift duration or exposure time, average number of encounters per hour, and the relative risk of a frontliner getting infected, which is proportional to the potential number of newly infected. Parameters used: $S_{0}=100, S_{\max }=100$, no protection,

The number of newly infected frontliners is directly proportional to the average number of encounters per hour (Table 1). Regardless of work shift duration, a hospital security guard or a triage nurse entertaining 120 persons per hour (1 patient every $30 \mathrm{~s}$ ) is at least 12 times more likely to get infected than a medical or radiation technologist encountering 10 patients per hour. A doctor conducting long-duration interviews and examinations on patients (20-80 persons per hour) is $2-8$ times more likely to get infected than, for example, a radiation technologist.

Regardless of the type of frontliner, a work shift duration of at least $10 \mathrm{~h}$ is at least 1.25 times more likely to be infected than that of $8 \mathrm{~h}$. The longer the work shift duration or exposure time, the higher the infection risk (Table 2).
$I_{0}=1$. a Relationship between average number of encounters per hour and expected number of new infecteds. b Relationship between work shift duration or exposure time and expected number of new infecteds

\subsection{Crowd density}

We define crowd density as the number of people in a room divided by the maximum capacity of the room. We can also define it as the average proportion of COVID-19-infected entities present within a $2-\mathrm{m}$ radius (minimum radius for social distancing) from the health care worker. The entity may be an infected patient (confirmed or not confirmed), or any object or surface in the immediate environment that contains SARS-CoV-2 virus particles. There is evidence that the virus particles stay as long as $3 \mathrm{~h}$ as aerosols and $72 \mathrm{~h}$ on plastic surfaces (van Doremalen et al. 2020).

Looking at the following figure (Fig. 2), crowd density acts as a fraction that modifies the risk of getting infected at all levels of encounter rates, from the laboratory (low encounter rate per hour) to the triage area (high encounter 
Table 1 Relative risk compared to 10 encounters per hour

\begin{tabular}{llll}
\hline $\begin{array}{l}\text { Number of } \\
\text { encounters per } \\
\text { hour }\end{array}$ & $\begin{array}{l}\text { Relative risk compared } \\
\text { to } 10 \text { encounters per } \\
\text { hour }\end{array}$ & $\begin{array}{l}\text { Assigned } \\
\text { risk } \\
\text { points }\end{array}$ & Examples \\
\hline 120 & $12 \times$ & 10 & Security guard, triage nurse, doctor doing long interviews \\
100 & $10 \times$ & 9.5 & \\
80 & $8 \times$ & 9 & A doctor conducting moderate- to long-duration interviews and examinations on several \\
60 & $6 \times$ & 8 & patients \\
40 & $4 \times$ & 7 & \\
30 & $3 \times$ & 6.5 & \\
20 & $2 \times$ & 6 & \\
10 & $1 \times$ & 5 & Radiologic technologist who does chest X-rays for persons-under-investigation (PUIs), \\
6 & $0.5 \times$ & 4 & or anyone who takes nasopharyngeal or oropharyngeal swab \\
3 & $0.25 \times$ & 3 & \\
2 & $0.167 \times$ & 2 & \\
1 & $0.0833 \times$ & 1 & Medical technologist who collects specimen only at certain timeslots, or any health \\
& & & worker with minimal patient interaction
\end{tabular}

${ }^{a}$ This is a sample qualitative point system that can be adjusted or modified depending on the situation

Table 2 Relative risk compared to an 8-h work shift duration or exposure time

\begin{tabular}{lll}
\hline $\begin{array}{l}\text { Work shift dura- } \\
\text { tion or exposure } \\
\text { time (h) }\end{array}$ & $\begin{array}{l}\text { Relative risk compared to an 8-h } \\
\text { work shift duration or exposure } \\
\text { time }\end{array}$ & $\begin{array}{l}\text { Assigned } \\
\text { risk } \\
\text { points }^{\mathrm{a}}\end{array}$ \\
\hline 30 & $3.75 \times$ & 10 \\
24 & $3 \times$ & 8 \\
12 & $1.5 \times$ & 6 \\
10 & $1.25 \times$ & 3 \\
8 & $1 \times$ & 2.5 \\
6 & $0.75 \times$ & 2 \\
4 & $0.5 \times$ & 1.5 \\
2 & $0.25 \times$ & 1 \\
1 & $0.125 \times$ & 0.5 \\
\hline
\end{tabular}

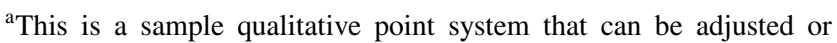
modified depending on the situation

rate per hour). A crowded place where social distancing is not highly implemented can initiate transmission of the disease. Having more space available for each patient, putting dividers between infected patients, and cleaning the workspaces more often lead to a lower crowd density. A lower crowd density implies that the frontliner is receiving a lesser fraction of the risk of infection. A higher crowd density increases the chance of being infected.

\subsection{Initial number of infected atients}

The effect of crowd density is so important that, even if ten COVID-19-infected patients enter the same room at the same time, the risk of the frontliner getting infected can be dramatically reduced by reducing the crowd density (Fig. 3).
For example, a health care worker in a room with crowd density of $10 \%$ is at least $95 \%$ less likely to be infected than a health care worker in a room with crowd density of $100 \%$. Moreover, as expected, the number of newly infected patients in a room is directly proportional to the infection risk faced by the frontliners (Fig. 3).

\subsection{Protection level}

Protection level is defined as the fraction of the risk being removed or mitigated by measures done by the health care worker or any other person. It has a minimum value of 0 and a maximum value of 1 . It can be observed in Fig. 4 that a $95 \%$ or better protection level significantly reduces infection risk. We can assign values which may be additive as shown in Table 3.

Regardless of the number of COVID-19 patients entering a given location at the same time, regardless of the average number of encounters per hour, and regardless of the work shift duration or exposure time, the protection level removes a substantial fraction of the risk faced by the health care worker (Fig. 4). In general, having PPEs confers protection towards the health care worker, but certain procedures, especially doing an endotracheal intubation for critically ill COVID-19 patients, exposes the health care worker to aerosolized particles.

However, the number of COVID-19 patients entering at the same time at a given place influences the level of protection needed (Fig. 5). For even an hour of exposure, when ten COVID-19 patients enter at the same time in the same place, the risk of getting infected with $70 \%$ level of protection is the same as the risk of getting infected when there is no PPE worn in a room with at most three COVID-19 patients. 


\section{Average number of encounters per hour $=1$}

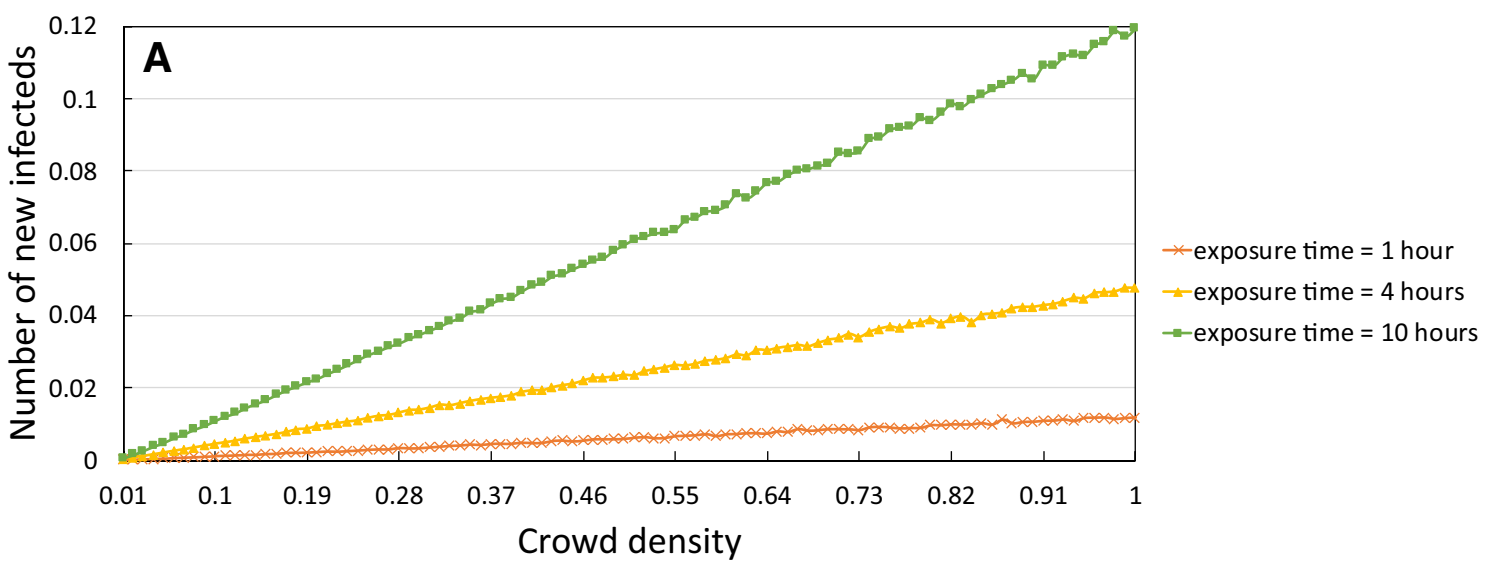

Average number of encounters per hour $=12$

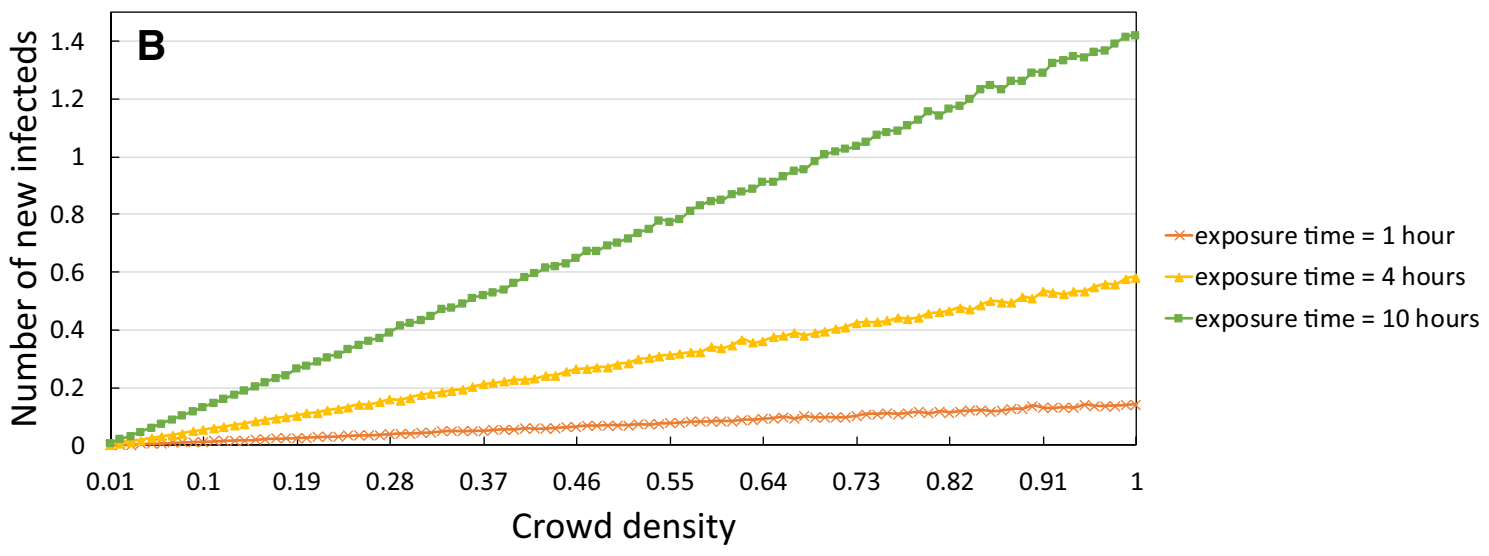

Average number of encounters per hour $=\mathbf{1 2 0}$

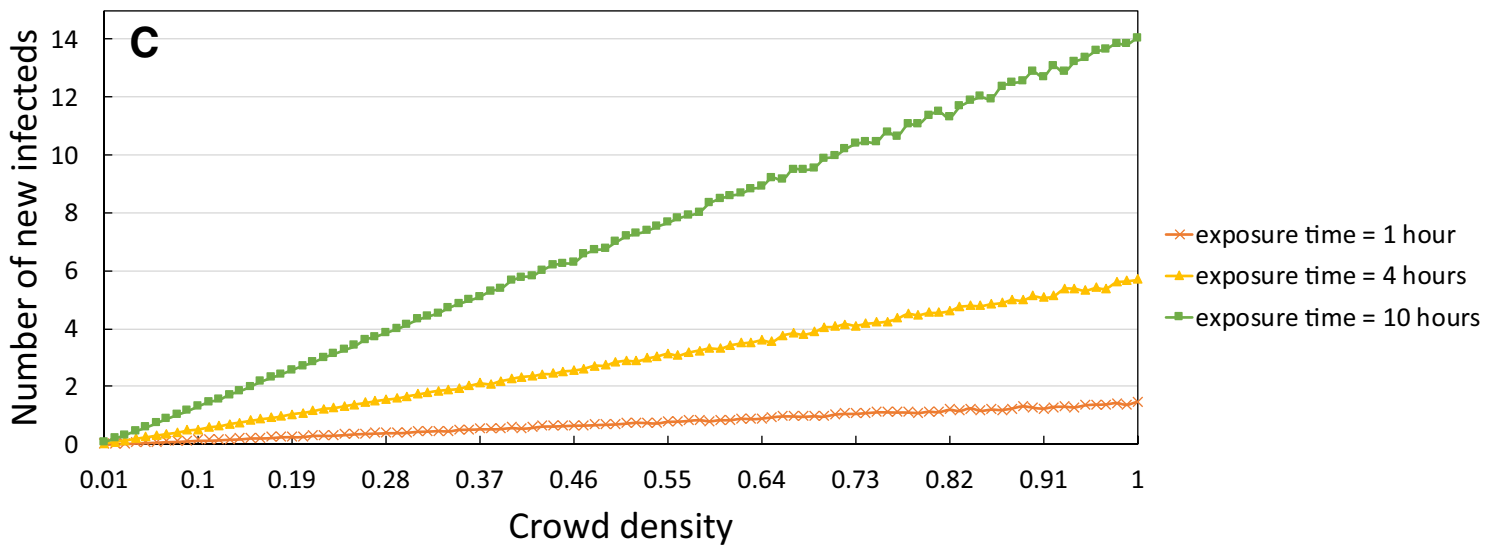

Fig. 2 Effect of crowd density on infection risk with varying number of encounters and exposure time (work shift duration). Parameters used: $S_{0}=100, S_{\max }=100$, no protection, $I_{0}=1$. Average number of encounters per hour $\mathbf{a}=1, \mathbf{b}=12, \mathbf{c}=120$

\subsection{Risk assessment}

From the previous tables and figures, we, therefore, propose an overall risk score to be used by each frontliner (Tables 4, 5). We define the overall risk score as a function of the expected number of new infected persons. If the overall risk score is less than 1.0, then there is a low risk that a person will become infected. An increase in the risk score is proportional to the increase in the number of persons expected to be infected. For example, 
Fig. 3 Risk of infection determined by the number of infected cases present in a room with the frontliners. Parameters used: $S_{\max }=100$, no protection, average number of encounters per hour $=120$, work shift duration or exposure time $=1 \mathrm{~h}$

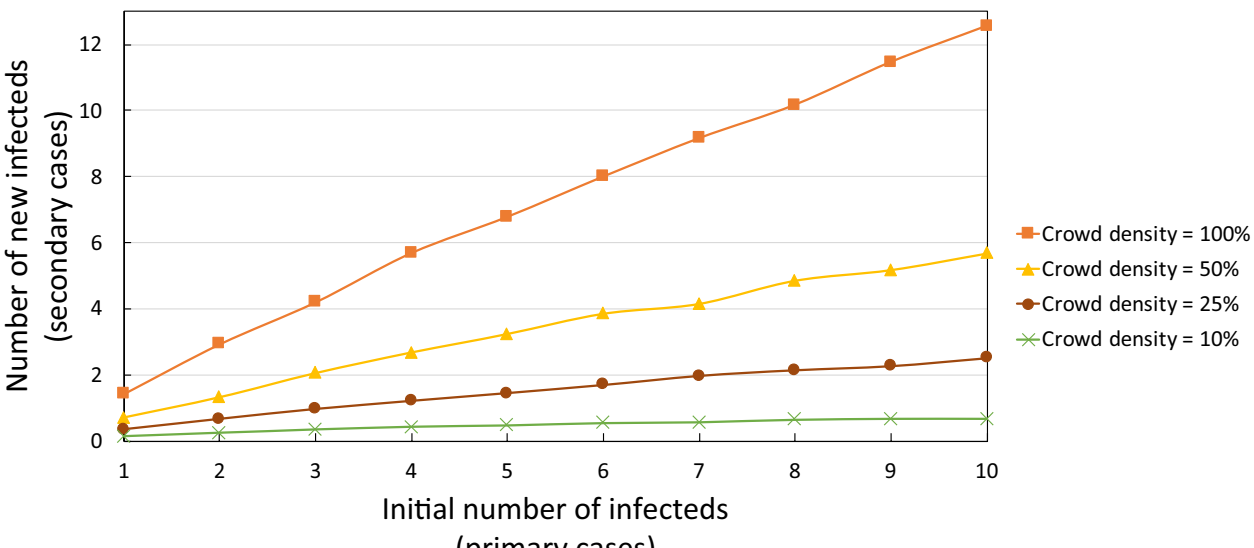

(primary cases) an overall risk score of 2.00 implies that there are two expected new infected persons. The maximum overall risk score is 10 (where Points encounter rate $=10$, Points $_{\text {duration }}=10$, Crowd Density $=1$, and Protection Level $=0$ ). The proposed formula is defined as:

Overall Risk Score $=\frac{\left(\text { Points }_{\text {encounter rate }}+\text { Points }_{\text {duration }}\right)}{2}$ $\times$ Crowd Density $\times(1-$ Protection Level $)$.

Analogous to the "low" and "high" risk assessments for health care providers of the United States Centers for Disease Control and Prevention (US CDC) (United States Centers for Disease Control and Prevention 2020), we likewise propose a categorization of "low risk" and "high risk". If the overall risk score is greater than or equal to 1.0, then there is a high risk of a person getting infected.

We can also add an intermediate category between "low" and "high" risks. A "moderate" risk category may be defined as those overall risk scores between 0.5 and 1 . By doing a Monte Carlo sensitivity analysis (5 million simulation runs), assuming each input has $10 \%$ error, the overall risk score has 0.45 standard error. To account for this uncertainty, overall risk scores between 0.5 and 1 can be classified under the qualitative category of Moderate Risk.

The overall risk score can be used to compare practices. Moreover, as the number of days the health care worker is doing his or her regular job related to a COVID-19 task, the risk of infection increases. The number of days can be scaled accordingly as exposure time.

From the simulations, we have proposed risk assessment criteria and several recommendations. Through these, governments and organizations can have insights on how to minimize or eliminate the transmission of SARS-CoV-2, especially in health care facilities. It is important to protect our health care workers as they are considered essential part of the health care capacity that can provide optimal care to the patients. To test the utility of our model, the model has been used in the Job Risk Profiling Calculator (https://datas tudio.google.com/s/iCAEymT7alg) that is used by private and public institutions in the Philippines.

All in all, based on the simulations, the following recommendations can be made:

- Decreasing the rate of patient encounters per frontliner, such as having multiple frontline triage nurses, multiple queues, multiple entrances, and proper referral systems, mitigates the risk of infection. Crowd density factor should always be considered. Having many COVID-19 patients in a room can render a protective measure relatively inadequate. It is recommended to have a quota on the number of COVID-19 patient encounters per duration of work shift. Telemedicine or online consultations can also be an effective control in reducing high risk frontliner-patient interaction (Shaikh 2015; Burton et al. 2012).

- Shorter work shift duration or exposure time reduces the risk faced by the frontliners, especially the security guard and the triage nurse. The protection level against SARS$\mathrm{CoV}-2$ transmission must be increased accordingly if shortening of work shift duration is not feasible.

- Increased spacing, frequent cleaning of work spaces, and compartmentalizing the rooms of patients in open space decrease the risk of infection not only for health care workers but also for the other patients who are COVID19 negative or non-person-under-investigation (PUI) (Nishiura et al. 2020).

- Personal Protective Equipment (PPE) plays a vital role in decreasing the risk of infection, but this protective factor can be overwhelmed by the sheer number of COVID-19 patients (whether confirmed or not). Hence, this should not be relied upon alone, and other structural factors, such as crowd density, be adjusted.

- Frontliners who are handling risky procedures such as endotracheal intubation (i.e., anesthesiologists) on critically ill COVID-19 patients (who are most likely to be 
Fig. 4 Effect of protection level on the reduction of infection risk. Parameters used: $S_{0}=100$, $S_{\max }=100$, no protection, $I_{0}=1$, average number of encounters per hour $=120$, exposure time $=1 \mathrm{~h}$. Initial number of infected $\mathbf{a}=1, \mathbf{b}=2, \mathbf{c}=5$, $\mathbf{d}=10$
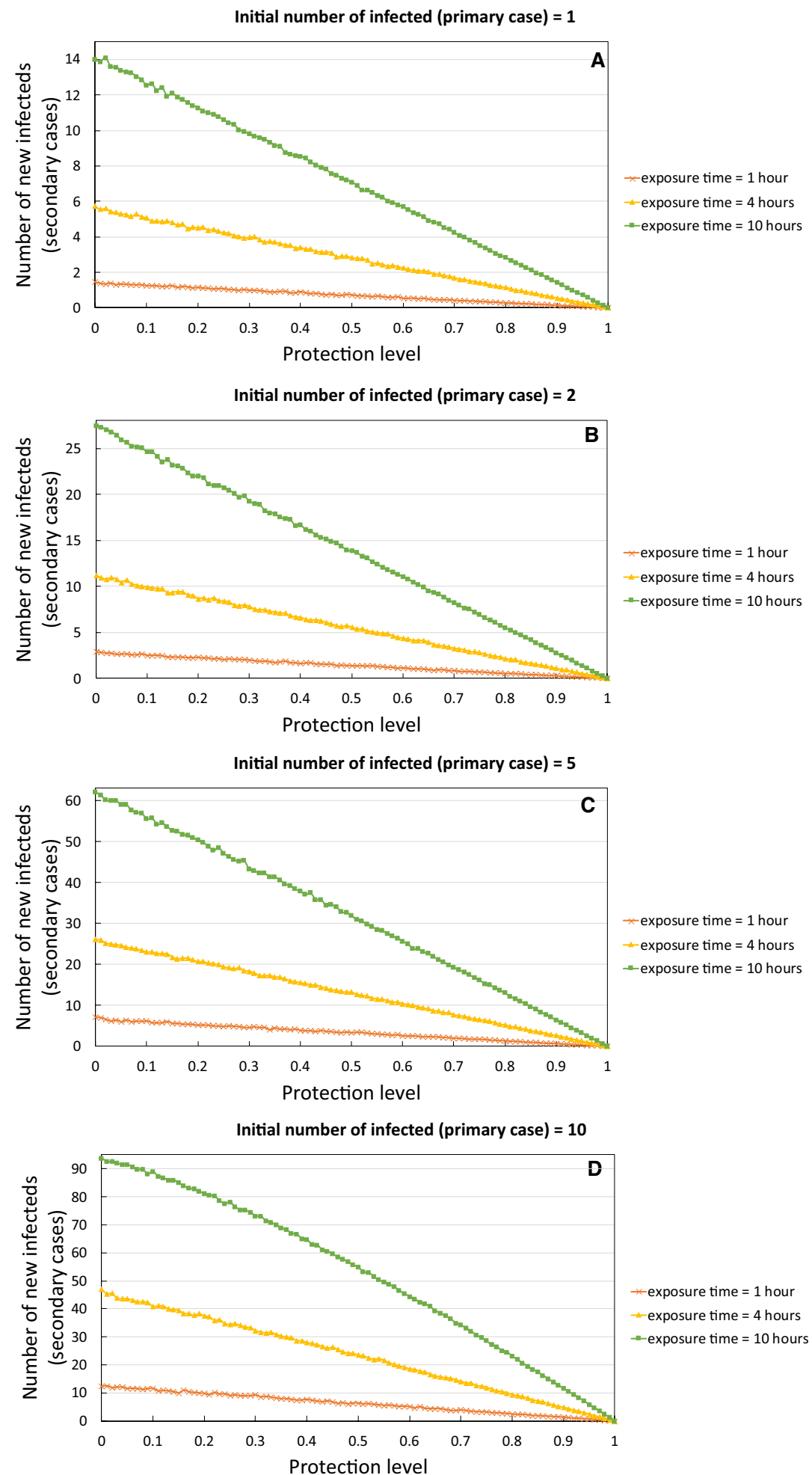

the most infectious), must be given extra protection, and hospital policies must minimize their duration of exposure and the number of patients they encounter or interact with per shift.
- Health care workers play a very important role in a community's battle against the medical effects of COVID-19. Decreasing the infection risks faced by each health care worker per day, coupled with superior health, well-being and welfare practices, will result in a robust health care 
Table 3 Examples of protection level points for every procedure and equipment worn

\begin{tabular}{|c|c|}
\hline Protection level points ${ }^{\mathrm{a}}$ & Description \\
\hline 0.00 & Having no personal protective equipment (PPE), not following hand hygiene \\
\hline$-0.50 \sim-0.60$ & $\begin{array}{l}\text { During an endotracheal intubation via direct laryngoscopy, where exposure } \\
\text { to aerosolized respiratory particles is at the maximum (e.g., for anesthesi- } \\
\text { ologists) }\end{array}$ \\
\hline$-0.40 \sim-0.50$ & Exposure to a coughing patient who is not wearing a mask \\
\hline$-0.30 \sim-0.40$ & Doing a nasopharyngeal or oropharyngeal swab on COVID-19 patients \\
\hline$-0.20 \sim-0.30$ & During an endotracheal intubation via video laryngoscopy \\
\hline$-0.20 \sim-0.30$ & Exposure to a coughing patient who is wearing a mask \\
\hline$+0.10 \sim 0.20$ & Wearing gloves \\
\hline$+0.20 \sim 0.30$ & Wearing surgical masks \\
\hline$+0.20 \sim 0.30$ & Strict compliance of hand hygiene \\
\hline$+0.30 \sim 0.40$ & Wearing N95 masks \\
\hline$+0.30 \sim 0.40$ & Having face and eye shields \\
\hline$+0.50 \sim 0.90$ & Wearing a full body biohazard suit \\
\hline$\approx 1.00$ & $\begin{array}{l}\text { Absolute protection; either being totally absent from the job or out of shift, } \\
\text { or being in full and functional PPE (N95 masks, face and eye shields, } \\
\text { gloves, biohazard suit); strict compliance with hand hygiene; having effec- } \\
\text { tive engineering controls }\end{array}$ \\
\hline
\end{tabular}

${ }^{\text {a }}$ This is a sample qualitative point system that can be adjusted or modified depending on the situation
Fig. 5 Effect of protection level on reducing the infection risk depending on the number of COVID-19 patients present. Parameters used: $S_{0}=100$, $S_{\max }=100$, no protection, $I_{0}=1$, average number of encounters per hour $=120$, exposure time $=1 \mathrm{~h}$

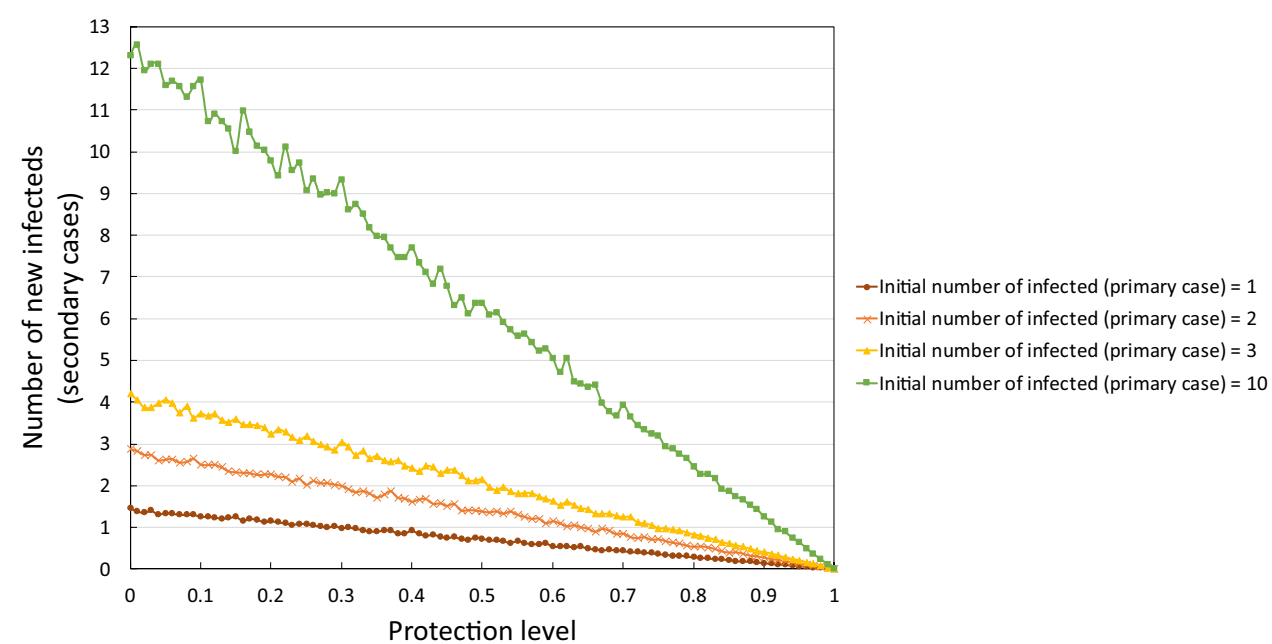

Table 4 Proposed risk assessment based on overall risk score

\begin{tabular}{ll}
\hline Overall Risk Score & Risk assessment \\
\hline 0 to less than 0.5 & Low risk \\
0.5 to less than 1.0 & Moderate risk \\
Greater than or equal to 1.0 & High risk \\
\hline
\end{tabular}

staff that can endure a long period of battle during this COVID-19 pandemic.

- Decreasing the infection risk discussed in this paper can also be extended to decreasing the infection risk of
non-COVID-19 patients present in a hospital. Moreover, the model and results presented here can be customized for other similar scenarios, such as identifying infection risk in public transportation, school classroom settings, offices, and mass gatherings.

- The recommendations in this paper is based on a theoretical model with parameters calibrated for COVID-19. The theoretical model and algorithm in this paper can be modified for other diseases. It is suggested to validate the results through experiments or cohort and case-control studies. 


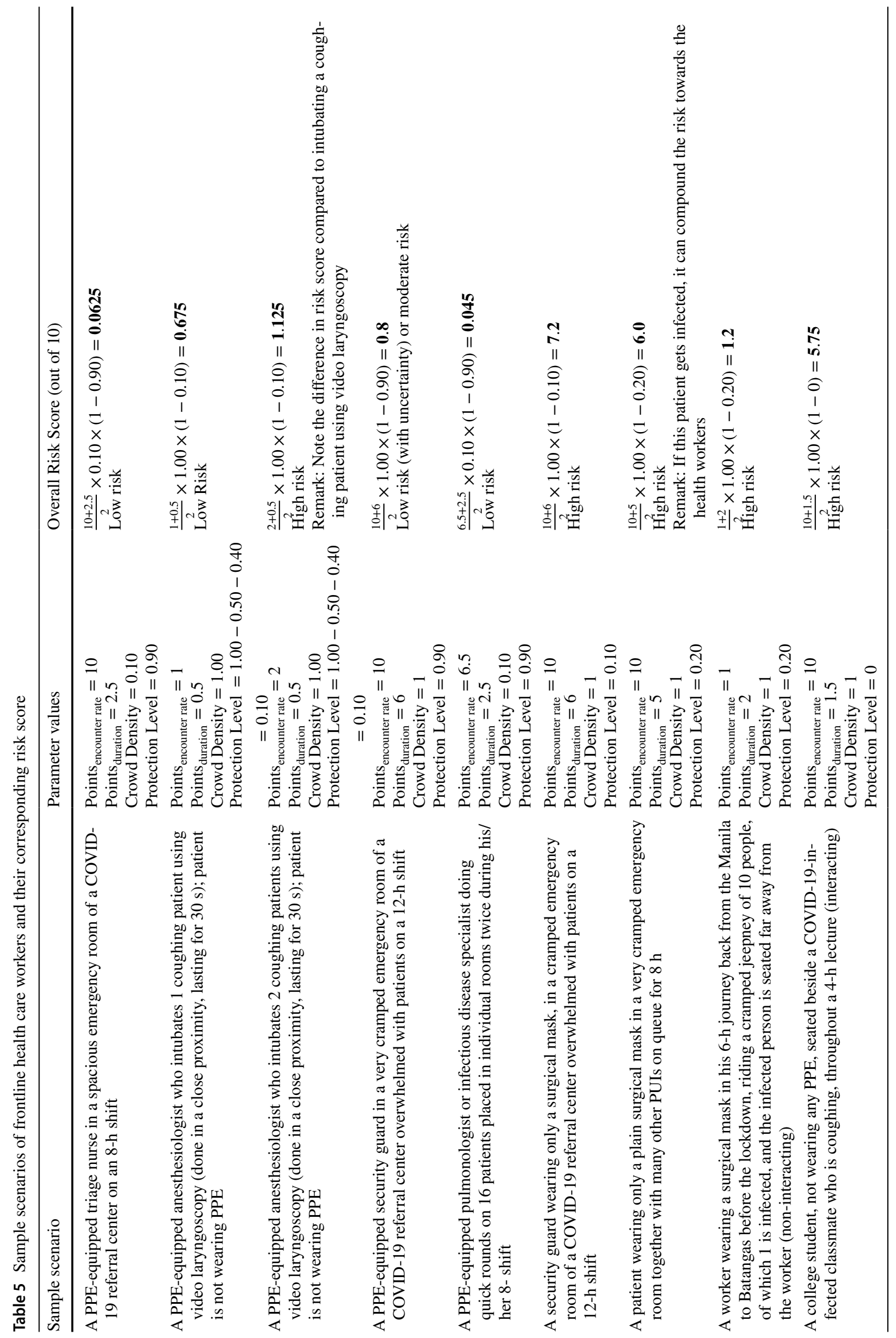




\section{Conclusion}

In this study, we formulated a mathematical model to calculate the risk of being infected in health care facilities. We considered the following factors: (1) the average number of encounters with a suspected COVID-19 patient per hour; (2) interaction time for each encounter; (3) work shift duration or exposure time; (4) crowd density, which may depend on the amount of space available in a given location; and (5) availability and effectiveness of protective gears and facilities provided for the frontline health care workers. A set of risk assessment criteria has been proposed based on the theoretical results to determine if a frontliner is facing low, moderate or high risk of infection.

Based on the simulations and risk assessment, several recommendations are suggested, namely (1) decrease the rate of patient encounter per frontline health care worker, e.g., maximum of three encounters per hour in a 12-h work shift duration; (2) decrease the interaction time between the frontline health care worker and the patients, e.g., less than 40 min for the whole day; (3) increase the clean and safe space for social distancing, e.g., maximum of $10 \%$ crowd density, and if possible, implement compartmentalization of patients; and/or (4) provide effective protective gears and facilities, e.g., 95\% effective, that the frontline health care workers can use during their shift.

Acknowledgement JFR is supported by The Abdus Salam International Centre for Theoretical Physics Associate Scheme, Trieste Italy.

\section{Compliance with ethical standards}

Conflict of interest The author declares that there are no conflicts of interest.

\section{Appendix: Methods}

The estimated values for the number of possible newly infected patients are generated using the Runge-Kutta 4 (RK4) Method of integration to solve the system of differential equations. We use the software (Berkeley Madonna for Mac ver.9.1.19). The differential equations are based on an S-E-I compartment model of disease transmission (See Fig. 6).

The model is described by the following system of differential equations:

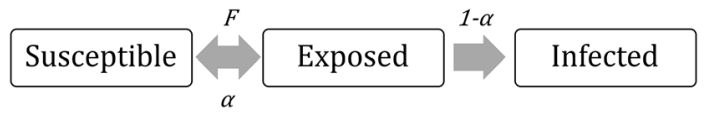

Fig. 6 An S-E-I compartment model of disease transmission $\frac{d S}{d t}=-F S+\alpha E$

$\frac{d E}{d t}=F S-\alpha E-(1-\alpha) E=F S-E$,

$\frac{d I}{d t}=(1-\alpha) E$,

where $S, E, I$ are the number of susceptible, exposed, and infected persons (in this case, frontliners); $F$ is the force of infection; $\alpha$ is the rate of an exposed individual becoming not infected (e.g., through handwashing or other protective measures); and $1-\alpha$ is the rate of getting infected.

The force of infection $F$ is defined as:

$F=\lambda \bullet I_{0}$,

where $\lambda$ is the effective transmission rate, and $I_{0}$ is the initial number of infected persons (or "the inoculum"), where the effective transmission rate $\lambda$ is defined as:

$\lambda=p \bullet \beta$,

where $\beta$ is the transmission risk or probability, and $p$ is the total contact rate. We cannot express $p$ as $I / N$, which is the ratio of the total number of infected persons to the total population in a given area because it assumes that everyone is homogenously distributed in a given place. Instead, we note that $p$ is in terms of the fraction of the initial number of susceptibles $\left(S_{0}\right)$ over the maximum number of susceptibles that a given area can accommodate $\left(S_{\max }\right)$, multiplied by the encounter ratio $\left(\frac{\mu}{\theta}\right)$, where $\mu$ is the average number of encounters per hour and $\theta$ is the threshold number of encounters per hour. Suppose $\theta=N$ per hour. If $\frac{\mu}{\theta}<1$ then we are sure that the frontliner has not yet encountered everyone in the room; if $\frac{\mu}{\theta}=1$ then there is a possibility that the frontliner already encountered everyone in the room; if $\frac{\mu}{\theta}>1$ then we are sure that the frontliner encountered a person in the room more than once (by Pigeonhole Principle). The ratio $\frac{S_{0}}{S_{\max }}$ can also be interpreted as the crowd density. The case where $S_{0}=S_{\max }$ and $\mu=\theta$ characterizes the usual wellmixed S-E-I model. The parameter $\beta$ (e.g., $\beta=0.2$ (Ferretti et al. 2020)) is assumed to be a function of the COVID-19 basic reproductive number (e.g., $R_{0}=3$ ) divided by the infectious period (e.g., $\tau=14$ ). We can also interpret $\frac{\mu}{\theta}>1$ as increasing the average nature of the reproductive number.

Therefore: 
$p=\frac{S_{0} \mu}{S_{\max } \theta}$

$\beta=\frac{R_{0}}{\tau}$,

$\lambda=\frac{S_{0} \mu R_{0}}{S_{\max } \theta \tau}$

$F=\frac{S_{0} \mu R_{0}}{S_{\max } \theta \tau} I_{0}$

which results in the following:

$\frac{d S}{d t}=-\left(\frac{S_{0} \mu R_{0}}{S_{\max } \theta \tau} I_{0}\right) S+\alpha E$,

$\frac{d E}{d t}=\left(\frac{S_{0} \mu R_{0}}{S_{\max } \theta \tau} I_{0}\right) S-\alpha E-(1-\alpha) E=\left(\frac{S_{0} \mu R_{0}}{S_{\max } \theta \tau}\right) S-E$, $\frac{d I}{d t}=(1-\alpha) E$

The goal of the study is to investigate the risk of individuals during one cycle of day job. The simulations should end before $48 \mathrm{~h}$ since the dynamics may already change as the newly infected person also becomes infectious.

The exposed $(E)$ class here, in contrast to the classical SEIR model, does not represent persons at the latency stage of the disease. Simply, E class here includes people who have been exposed with the virus (e.g., from respiratory droplets) but the persons can be unexposed through protections (e.g., through washing hands and wearing of face masks). Incubation period is not included because the goal of the study is only for short duration (less than $48 \mathrm{~h}$ ). The I compartment is only for counting how many new individuals have been infected, and is not intended to reflect feedback loop leading to a community outbreak. The paper is for risk assessment and not intended to simulate epidemics in the whole community. 
The following is the Berkeley Madonna code:

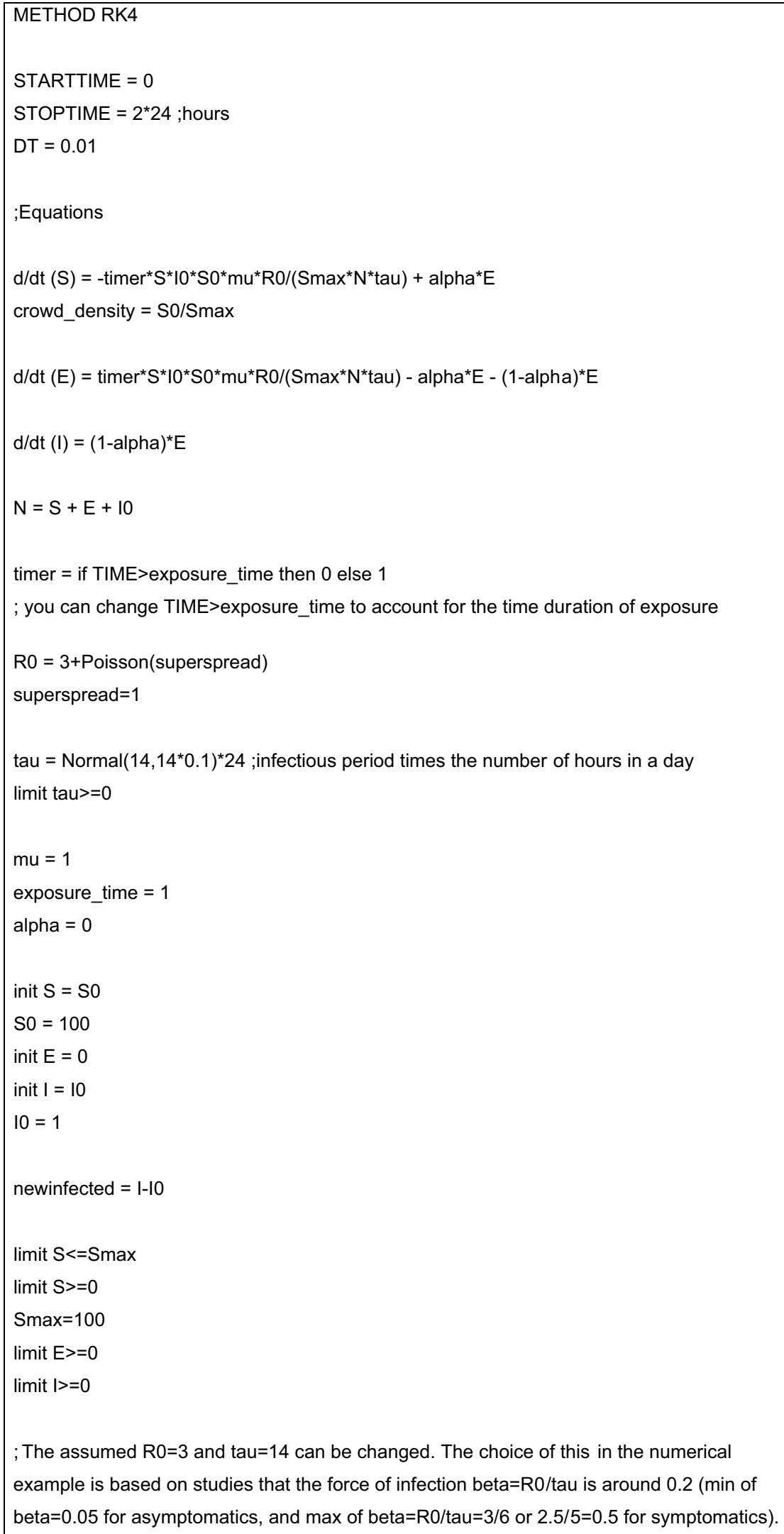




\section{References}

Anderson RM et al (2020) How will country-based mitigation measures influence the course of the COVID-19 epidemic? Lancet 395:931-934

Baticulon R (2020) OPINION: the Philippine healthcare system was never ready for a pandemic. CNN Philipiines. https://cnnph ilippines.com/life/culture/2020/3/20/healthcare-pandemic-opini on.html. Accessed 22 Mar 2020.

Burton SH, Tanner KW, Giraud-Carrier CG (2012) Leveraging social networks for anytime-anyplace health information. Netw Model Anal Health Inform Bioinform 1:173-181

Choudhury A, Kaushik S, Dutt V (2018) Social-network analysis in healthcare: analysing the effect of weighted influence in physician networks. Netw Model Anal Health Inform Bioinform 7:17

Cortez MJV et al (2017) From epigenetic landscape to phenotypic fitness landscape: evolutionary effect of pathogens on host traits. Infect Genet Evol 51:245-254

Dayrit MM et al (2018) The Philippines health system review. World Health Organization. Regional Office for South-East Asia. https ://apps.who.int/iris/handle/10665/274579

Ferguson NM et al (2020) Impact of non-pharmaceutical interventions (NPIs) to reduce COVID19 mortality and healthcare demand. Imperial College COVID-19 Response Team. https:// www.imperial.ac.uk/media/imperial-college/medicine/sph/ide/ gida-fellowships/Imperial-College-COVID19-NPI-modelling16-03-2020.pdf

Ferretti L et al (2020) Quantifying SARS-CoV-2 transmission suggests epidemic control with digital contact tracing. Science 368:eabb6936

Italy Coronavirus Death Toll Overtakes China (2020) BBC. https:// www.bbc.com/news/world-europe-51964307. Accessed 20 Mar 2020 .
Nishiura $\mathrm{H}$ et al (2020) Closed environments facilitate secondary transmission of coronavirus disease 2019 (COVID-19). medRxiv. https ://www.medrxiv.org/content/10.1101/2020.02.28.20029272v1

Novel Coronavirus Situation Reports (2020) World Health Organization (WHO). https://www.who.int/emergencies/diseases/novel -coronavirus-2019/situation-reports

Panela S (2020) Metro Manila coronavirus cases could peak mid-April if not contained, math model shows. Rappler. https://www.rappl er.com/nation/255060-math-model-coronavirus-cases-metro -manila-could-peak-mid-april-not-contained. Accessed 22 Mar 2020.

Rabajante JF (2020) Insights from early mathematical models of 2019nCoV acute respiratory disease (COVID-19) dynamics. J Environ Sci Manag 23-1:1-12

Shaikh A (2015) The impact of SOA on a system design for a telemedicine healthcare system. Netw Model Anal Health Inform Bioinform 4:15

United States Centers for Disease Control and Prevention (2020) Interim operational considerations for public health management of healthcare workers exposed to or with suspected or confirmed COVID-19: non-U.S. Healthcare Settings. United States Centers for Disease Control and Prevention. https://www.cdc.gov/coron avirus/2019-ncov/hcp/non-us-settings/public-health-management -hcw-exposed.html

van Doremalen N et al (2020) Aerosol and Surface Stability of SARSCoV-2 as Compared with SARS-CoV-1. N Engl J Med. https:// doi.org/10.1056/nejmc2004973

Publisher's Note Springer Nature remains neutral with regard to jurisdictional claims in published maps and institutional affiliations. 